\title{
Quantification of Sympathetic Transduction in Type 2 Diabetes Patients
}

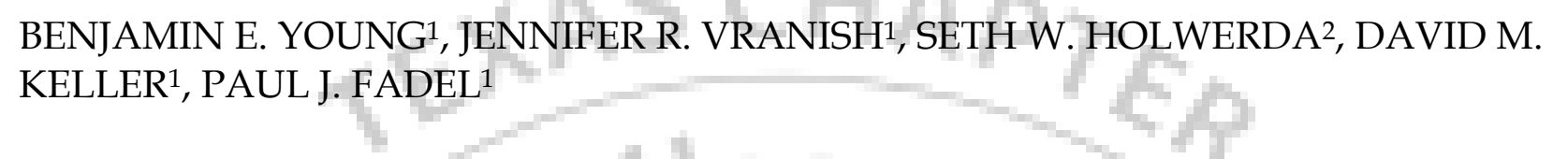

${ }^{1}$ Human Neural Cardiovascular Control Lab; Department of Kinesiology; University of Texas at Arlington; Arlington, TX

2Translational Vascular Physiology Lab; Department of Health and Human Physiology; University of Iowa; Iowa City, IA

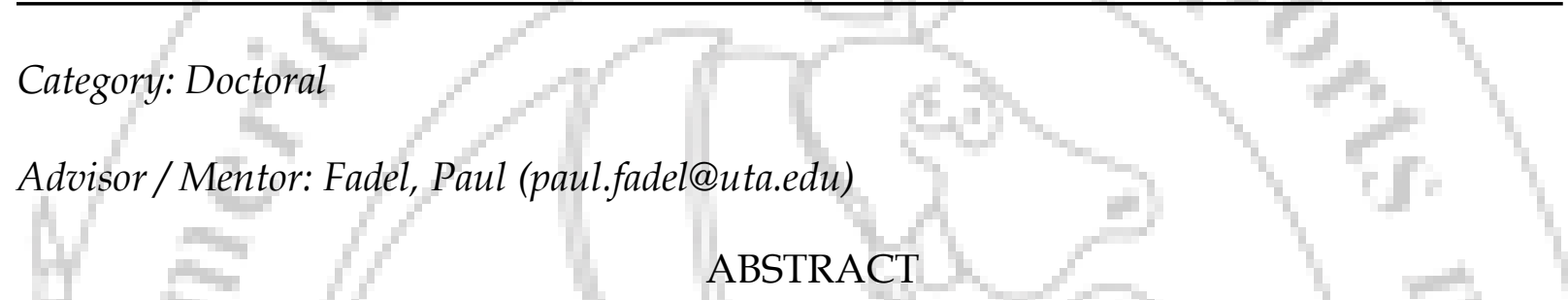

Type 2 Diabetes patients (T2D) have been shown to have greater alpha-adrenergic sensitivity. How this impacts the transduction of muscle sympathetic nerve activity (MSNA) to arterial blood pressure under resting conditions using spontaneous fluctuations in MSNA, as well as during stressors known to elicit sympatho-excitation (e.g., cold pressor test (CPT)) is unclear. PURPOSE: We tested the hypothesis that T2D patients would exhibit greater sympathetic transduction compared to age- and BMI-matched, healthy controls. METHODS: MSNA (microneurography), heart rate (ECG), and beat-to-beat arterial blood pressure (finger photoplethysmography) were continuously recorded during a 10 minute baseline period, and in response to a 2-minute CPT in six T2D patients and six age- and BMI-matched, healthy controls (CON).To quantify sympathetic transduction at rest, normalized burst heights were divided into four quartiles (smallest to largest), related to the corresponding peak change in mean arterial pressure (MAP) within those quartiles and a slope was determined. To quantify sympathetic transduction in response to a stressor, the change in MAP was related to the change in MSNA from rest to the last minute of CPT. RESULTS: There were no differences in resting sympathetic transduction between groups (CON slope: $0.0103 \pm 0.0023 \mathrm{mmHg} / \mathrm{AU}$, T2D slope: $0.0095 \pm 0.0016 \mathrm{mmHg} / \mathrm{AU} ; \mathrm{p}=0.78)$. Indeed, signal averaging of MSNA bursts indicated a similar peak increase in blood pressure in CON $(+4.2 \pm 0.6 \mathrm{mmHg})$ and T2D $(+4.0 \pm 0.9 \mathrm{mmHg})(\mathrm{p}=0.66)$. Although the peak increase in blood pressure to CPT tended to be higher in T2D (T2D: +31.6 $\pm 3.4 \mathrm{mmHg}$, CON: $+21.4 \pm 3.7 \mathrm{mmHg}$; $=0.096$ ), the $\triangle \mathrm{MAP} / \Delta$ MSNA relationship during CPT was not different between groups (CON: 0.4158 \pm 0.21 , T2D: $0.1862 \pm 0.05 ; \mathrm{p}=0.36$ ). CONCLUSIONS: Despite clear sympathetically-mediated increases in blood pressure in T2D patients and healthy CON subjects both at rest and during the CPT, neither of the methodologies used to estimate sympathetic transduction, with respect to changes in arterial blood pressure, detected group differences.

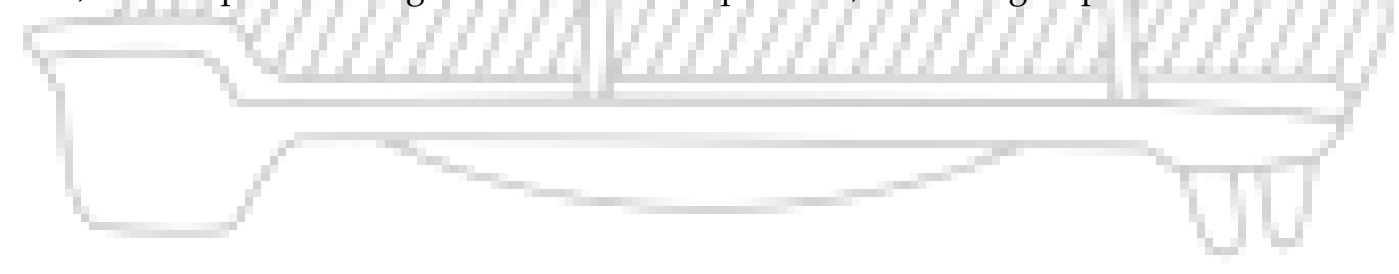

